\title{
Stendhal, Balzac, Dumas. Un récit romantique?, sous la direction de Lise Dumasy, Chantal Massol, Marie- Rose Corredor
}

\section{Michel Arrous}

\section{OpenEdition}

Journals

Édition électronique

URL : http://journals.openedition.org/studifrancesi/7595

DOI : 10.4000/studifrancesi.7595

ISSN : 2421-5856

Éditeur

Rosenberg \& Sellier

\section{Édition imprimée}

Date de publication : 1 décembre 2009

Pagination : 636-637

ISSN : 0039-2944

\section{Référence électronique}

Michel Arrous, «Stendhal, Balzac, Dumas. Un récit romantique?, sous la direction de Lise Dumasy, Chantal Massol, Marie-Rose Corredor », Studi Francesi [En ligne], 159 (LIII | III) | 2009, mis en ligne le 30 novembre 2015, consulté le 08 janvier 2021. URL : http://journals.openedition.org/studifrancesi/7595 ; DOI : https://doi.org/10.4000/studifrancesi.7595

Ce document a été généré automatiquement le 8 janvier 2021.

\section{cc) (†) $\odot$}

Studi Francesi è distribuita con Licenza Creative Commons Attribuzione - Non commerciale - Non opere derivate 4.0 Internazionale. 


\title{
Stendhal, Balzac, Dumas. Un récit romantique?, sous la direction de Lise Dumasy, Chantal Massol, Marie- Rose Corredor
}

\author{
Michel Arrous
}

\section{RÉFÉRENCE}

AA. VV., Stendhal, Balzac, Dumas. Un récit romantique?, sous la direction de Lise DUMASY, Chantal MASSOL, Marie-Rose CORREDOR, Toulouse, Presses Universitaires du Mirail, 2006, pp. 321.

1 L'intérêt de ce recueil des actes d'un colloque organisé à Grenoble III en novembre 2001 tient sans aucun doute à l'association de trois romanciers, romantiques chacun à leur façon, que la tradition sépare. Les intervenants ont tenté de répondre à deux questions liées: y a-t-il un récit romantique, et quel est le rôle des singularités propres à chaque auteur? Première évidence: ce qui rapproche Stendhal, Balzac et Dumas, c'est l'Histoire, consubstantielle au récit romantique. L'étude de la représentation romanesque des sociétés secrètes, dans Les Mohicans de Paris, l'Histoire des Treize et L'Envers de l'histoire contemporaine, et surtout l'évaluation des procédés narratifs (l'envers, le retardement, le dévoilement du secret, l'opposition du visible et du caché) qu'implique ce motif romanesque, auxquelles se livre Sarah MOMBERT (pp.21-31), permettent de vérifier l'hypothèse selon laquelle les «mystères du dessous»-l'expression est de Dumas révèlent ceux $\mathrm{du}$ dessus. C'est ce même double fond, cet univers occulte de la conspiration dans le Paris fantasmé par Balzac et Dumas, un Paris qui est un protagoniste à part entière, que Cécile MEYNARD explore en retraçant sa généalogie à partir du motif de la prairie chez Cooper (Les Mystères de Paris selon Balzac et Dumas: d'un motif romanesque traditionnel à la trame d'un récit romantique, pp.33-52). S'inspirant de 
Curtius, Anne-Marie CALLET-BIANCO (Un projet romantique: la quête de la totalité chez Balzac et Dumas, pp. 53-67) reprend l'idée de totalité qui caractérise l'œuvre de Balzac animé par la volonté de rendre compte d'un tout. A.-M. Callet-Bianco a le mérite de mettre en évidence cette même volonté chez Dumas, surtout à partir des Trois Mousquetaires: comme Balzac, Dumas ambitionne de construire une Histoire totale et de mettre en scène la totalité de la société. Le jeu intertextuel auquel se livrent Stendhal et Balzac à partir de 1832 offre à Hélène SPENGLER (pp. 70-99) l'occasion de montrer comment, dans Massimila Doni, l'imaginaire italien du second se nourrit de l'œuvre du premier, surtout avant 1836. À partir de Vanina Vanini, Un Épisode sous la Terreur et La Rose rouge, récits courts qu'unissent leur date et leur climat d'ensemble, Michel AUTRAND (pp. 101-115) cherche à vérifier si le récit romantique des années 1830 est bien, selon la définition qu'Henri Lemaître donnait du romantisme, «une fatalité inscrite dans une dynamique intérieure irrésistible, dont la source est dans les instincts originels et irrationnels de la nature humaine». On lira avec profit la fine analyse des éléments du cadre aussi bien que celle des modes de narration (un style «voyant, appuyé, affiché», des interventions directes nombreuses). Dans "Armance", "Le Lys dans la vallée" et la tradition du roman sentimental (pp. 119-136), Brigitte LOUICHON s'interroge sur les rapports qu'entretiennent ces deux œuvres avec la poétique et la typologie des formes du roman sentimental, de La Nouvelle Héloïse à Volupté. Béatrice DIDIER (pp.137-147) a choisi de s'intéresser au récit musical - biographie chez Stendhal, fiction chez Balzac (Sarrasine, Gambara) - afin d'étudier les différents procédés utilisés dans le montage du récit (effets de réel, digressions, discussions, intrusions du fantastique). Revenant sur la «question du style indirect libre» chez Stendhal et Balzac, Michel BRIX (pp. 149-161) rappelle que son utilisation vise à «mettre en procès le réalisme esthétique, c'est-à-dire la notion même du vrai littéraire», particulièrement chez Stendhal qui semble annoncer Flaubert déclarant: «Il n'y a pas de Vrai! Il n'y a que des manières de voir». Le long préambule de Ferragus, souvent assimilé à du discours auctorial, relèverait du discours indirect et illustrerait «l'écart séparant les choses elles-mêmes et les impressions nées des choses». Chez Balzac, comme chez Stendhal et sans doute chez Dumas, il n'y a pas de vision totalisante du réel. Vittorio FRIGERIO, qui revient sur la question initiale: Stendhal et Dumas évidemment romantiques? (pp.163-178), vérifie ces axiomes supposés incontestables que sont la rapidité et la légèreté (d'après la liste de valeurs à sauver établie par Calvino). Chez Stendhal, la rapidité, c'est l'usage répété de l'élision et la vitesse de l'action; chez Dumas, c'est la rapidité du débit, sa prolifération, sans réticences ni élisions. Quant à la légèreté - à ne pas confondre avec la désinvolture c'est, toujours selon Calvino, «une attention à l'infiniment petit», à l'individu plutôt qu'à l'ensemble, qui n'exclut pas la réflexion. À ce niveau, la distinction entre les styles est évidente: légèreté chez Stendhal, "pesanteur incroyablement alerte» chez Dumas. C'est sur l'usage des différents types de l'ellipse, plus particulièrement terminale, que se penche Jacques DÜRRENMATT (pp.179-192) en comparant l'usage qu'en font Dumas dans Marie, Balzac dans L'Élixir de longue vie et Stendhal dans Le Philtre. Énigmatique chez Stendhal dont le lecteur risque d'être désarçonné, privilégiant la recherche de l'effet narratif chez Dumas, alors que Balzac pousse son lecteur à suppléer à certaines insuffisances narratives. Autre caractéristique du récit romantique: les ruptures et les interruptions qui le traversent et désorganisent sa continuité. Pierre LAFORGUE (pp. 193-202) en analyse les effets à partir de quelques exemples tirés de La Chartreuse de Parme (suspens, stase, retardement, interruption, coupure) et d' Illusions perdues (l'intercalation de l'exposé qui vient interrompre la séquence de la promenade d'Ève et 
David): «À la différence de Stendhal qui se livre à des dynamitages narratifs ponctuels, Balzac, lui, agit à une bien plus vaste échelle». C'est encore des formes narratives ou plutôt des stratégies visant à faire accepter les longueurs que se préoccupent Aude DÉRUELLE (La question des longueurs (Stendhal, Balzac, Dumas), pp. 203-217) et Christèle COULEAU (Quand le roman parle à son lecteur. Stratégies du discours auctorial chez Balzac, Dumas, Stendhal, pp. 219-232). Si les longueurs ont été souvent condamnées, par les critiques ou les romanciers eux-mêmes, elles n'en sont pas moins utiles puisqu'elles permettent au roman de «dire le savoir». Chez Balzac, le discours savant «fait partie intégrante de l'entreprise de légitimation du roman»; chez Dumas, il s'agit de transmettre au lecteur un savoir historique; pour sa part, Stendhal privilégie la fonction du mimétique (esthétique du «miroir») imposée, comme souvent chez Balzac, par la volonté de témoigner d'un monde qui s'efface. Ch. Couleau s'est intéressée aux stratégies contrastées $\mathrm{du}$ discours auctorial mises en place par les trois romanciers, commentaires didactiques, explicatifs et idéologiques, adresses a lecteur, et même interventionnisme arbitraire et ironique chez Stendhal.

2 La dernière partie («Histoire, société, récit») s'ouvre par une intervention au titre accrocheur: L'Histoire à l'estomac. Corps, société, pouvoir dans le récit romantique (pp. 235-249), dans laquelle Jean-Marie Roulin, attentif à la sémiotisation du corps dans La Chartreuse de Parme (la caricature du gros archiduc), Les Chouans (le cadavre dont le tatouage signe l'appartenance politique) et La Reine Margot (Charles IX atteint dans son corps), montre clairement que «le récit romantique introduit le corps comme un véhicule du sens». Dans Trois romantiques face à l'Histoire: Stendhal, Balzac, Dumas, Isabelle DURAND-LE GUERN (pp. 265-281) retrace les manières d'appréhender l'Histoire dans Le Colonel Chabert, La Chartreuse de Parme et Le Comte de Monte-Cristo. Aucun n'étant strictement un roman historique, «l'événement historique est toujours abordé de biais» dans le cadre d'un processus de mythification (du personnage de Napoléon). L'Histoire joue un rôle de déclencheur et de catalyseur. De plus, un des enjeux du récit, et non le moindre, est de proposer une nouvelle lecture de la Restauration et de la monarchie de Juillet en révélant l' «envers de l'histoire contemporaine». Dans Raison et déraison vendettales dans "La Vendetta" de Balzac et "Les Frères corses" de Dumas (pp. 265-281), Claudie BERNARD distingue passion vindicative (réaction individuelle), système vindicatoire (réaction médiate) et punition (exercée par le supérieur) à l'œuvre dans ces deux récits qui mettent en scène l'extinction de la vendetta au profit de l'institution judiciaire. Adoptant une position non prescrite par le texte du roman, Constanze BAETHGE et Jacques DUBoIs (pp. 283-299) abordent la question de l'érotique et de la politique dans La Chartreuse de Parme et La Cousine Bette, œuvres fort distantes apparemment mais dont la configuration historique est proche puisqu'il s'agit dans les deux cas d'une société bloquée, par le biais d'une enquête sur la situation et la figuration des personnages féminins, Clélia et Valérie Marneffe. Une nouvelle lecture est proposée - que J. Dubois a développé depuis dans Stendhal, une sociologie romanesque (2007) - qui pousse à s'interroger sur les ellipses et les silences du travail fictionnel. L'analyse met non seulement en évidence les liens qu'entretient le roman avec l'Histoire, mais démontre aussi que le régime masculin, supposé dominer dans l'ordre des personnages, est contesté par de «jeunes conquérantes, prêtes à se perdre pour gagner». L'Histoire et la société jouent pleinement leur rôle dans le roman romantique sous bien des formes. Alexandre PÉRAUD (Sociopoétique de la rumeur dans "Lucien Leuwen", "La Maison Nucingen" et "Le Comte de Monte-Cristo", pp. 301-317) a choisi l'une d'entre 
elles, la rumeur ou, selon Stendhal, «la tyrannie de l'opinion», dans laquelle on peut voir une manifestation de la sociabilité bourgeoise qui menace l'individu. Dantès et Nucingen manipulent la rumeur pour se protéger des attaques du monde et parvenir à leurs fins; Lucien, quant à lui, adopte une stratégie défensive fort maladroite.

3 Des réflexions de qualité et souvent convergentes qui, pour la plupart, répondent précisément à la première question, mais délaissent quelque peu la seconde: y a-t-il un récit romantique ou, pour le dire autrement, les diverses formes narratives étudiées permettent-elles d'établir un modèle commun? 